\title{
COVID-19 and the liver-related deaths to come
}

Jean-Michel Pawlotsky ${ }^{1,2}$

Coronavirus disease 2019 (COVID-19) itself and/or the use of hepatotoxic drugs might negatively affect the course and management of patients with pre-existing chronic liver diseases. However, the greatest effect of COVID-19 on liver diseases will be indirect and delayed, resulting from the impending global economic crisis.

Coronavirus disease 2019 (COVID-19) was first reported in December 2019 in Wuhan, China and was considered harmless by many observers. As of May 30th 2020, according to the COVID-19 Dashboard by the Center for Systems Science and Engineering at Johns Hopkins University, USA, six million people worldwide have been diagnosed with COVID-19 and more than 365,000 of them have died from its complications. Meanwhile, the world economy is collapsing.

COVID-19, which is caused by severe acute respiratory syndrome coronavirus 2 (SARS-CoV-2), was initially thought to present essentially as an upper and/or lower respiratory tract infection. We now know that SARS-CoV-2 induces a systemic disease attacking many organs, potentially causing major damage, including mortality, and long-term sequelae. Surprisingly, the liver seems to be relatively spared by the virus. Indeed, apart from patients with the most severe forms of the disease resulting from so-called cytokine storm, who die from multivisceral deficiencies including the liver, manifestations of the infection in the liver are generally mild and transient and have no effect on the course of COVID-19 disease.

Studies based on single-cell RNA sequencing in healthy liver tissues suggest that the SARS-CoV-2 cellular receptor, angiotensin-converting enzyme 2 (ACE2), is present mainly on cholangiocytes, whereas expression is 20-fold lower in hepatocytes and ACE2 is not expressed in Kupffer cells or intrahepatic immune cells $^{1,2}$. The low or lack of expression of ACE2 in hepatocytes explains that SARS-CoV-2 does not actually cause viral hepatitis. COVID-19 is generally associated with mild-to-moderate elevations of aspartate aminotransferase (AST) and, to a lower extent, alanine aminotransferase (ALT) levels, which are more frequent in patients with severe disease than in those with a benign outcome ${ }^{3}$. Total bilirubin levels can also be slightly elevated, whereas alkaline phosphatase and $\gamma$-glutamyltransferase elevations have been rarely reported ${ }^{3}$. Nonspecific histological changes in the liver have been described in small autopsy series, ranging from moderate microvesicular steatosis with mild, mixed lobular and portal activity to focal necrosis, with detection of the virus in the liver in some cases ${ }^{4}$.

Changes in liver biochemistry might result from the systemic inflammatory response, pneumonia-induced hypoxaemia and/or drug-induced hepatic injury, especially in patients with severe forms of COVID-19 requiring hospitalization. The frequency of abnormal liver tests was reported to increase during hospitalization, possibly owing to the administration of hepatotoxic drugs, including antibiotics, nonsteroidal anti-inflammatory drugs, herbal products and antiviral agents, such as ribavirin, interferons, or the fixed-dose combination of lopinavir and ritonavir ${ }^{5}$. Elevations in aminotransferase levels have also been occasionally reported with other drugs used in clinical trials in patients with COVID-19, including chloroquine and hydroxychloroquine, azithromycin, remdesivir, favipiravir, camostat or tocilizumab. However, liver test abnormalities disappear when the infection recovers and/or when hepatotoxic drugs are removed.

Patients with chronic liver disease do not seem to be at greater risk of acquiring the infection than other individuals in the general population, but the potential onset of COVID-19 in these patients raises two questions: will patients with chronic liver disease develop a more severe form of COVID-19; and will COVID-19 aggravate the course of their liver disease and induce liver-related mortality? One study has suggested that 65 patients with non-alcoholic fatty liver disease (NAFLD) related to the metabolic syndrome were four times more likely to develop severe manifestations of COVID-19 than 65 patients without NAFLD, regardless of the presence of diabetes 5 . However, it is unclear whether NAFLD itself plays a part, rather than the associated metabolic comorbidities that include obesity, diabetes and hypertension, which are well-known risk factors for developing severe COVID-19. Patients with cirrhosis seem to be at greater risk than other individuals for severe COVID-19 and the occurrence of complications, including mortality, regardless of the aetiology 


\section{Box 1 |COVID-19 and liver-related mortality}

Coronavirus disease 2019 (COVID-19) has a modest effect on the liver, and liver-related mortality is unusual during the course of the disease, except in patients with severe pulmonary disease who die from multivisceral deficiencies including the liver or in patients with pre-existing advanced liver disease.

Most COVID-19-induced liver-mortality will be delayed, resulting from deferred care for liver diseases, reduced funding for public health interventions and the global economic crisis, which will lead to increases in alcohol and drug use and in blood-borne virus transmissions, while access to care and funding are reduced.

Prevention of liver-related mortality following the COVID-19 epidemic will require rapid resumption of care activities, in particular for patients with advanced cirrhosis or hepatocellular carcinoma and for those requiring liver transplantation, a massive investment in viral hepatitis elimination efforts and courageous economic and social measures to mitigate the health consequences of the coming economic crisis.

of liver disease ${ }^{6,7}$. The risk could also be increased in individuals with decompensated cirrhosis, but few cases have been reported. Data from registries from a large, multicentre international cohort of patients with chronic liver disease suggest that patients with compensated cirrhosis are at increased risk of decompensation and death during the course of COVID-19, even in the absence of respiratory symptoms ${ }^{8}$. The contribution of thromboembolic disorders to liver injury in patients with the most severe forms of COVID-19 has been suggested ${ }^{4}$. Such thromboembolic events might expose patients with pre-existing chronic liver disease to serious hepatic complications.

As a result of lockdowns and suspension of usual clinical care activities for the benefit of patients with COVID-19, the SARS-CoV-2 pandemic is having a major effect on the management of patients with chronic liver diseases, in particular those with cirrhosis, hepatocellular carcinoma and in liver transplantation programmes. The feared risk of acquiring COVID-19 at the hospital also prevented many patients from being appropriately managed by hepatology teams. The effect of the pandemic on care of patients with cirrhosis was anticipated to follow three waves: first, an intense period with prioritized high-acuity care with delayed elective procedures and routine care during physical distancing; then a challenging 'return to normal' following the end of physical distancing, with increased emergent decompensations, morbidity and systems of care overwhelmed by the backlog of deferred care; and finally, a protracted period of suboptimal outcomes characterized by missed diagnoses, progressive disease and loss to follow-up 9 The COVID-19 pandemic will also negatively affect the care and management of patients with hepatocellular carcinoma, generating delayed diagnosis, deferred treatment (including medical and surgical, such as access to liver transplantation), loss to follow-up and, ultimately, increased mortality.

Liver transplantation has been challenging during the COVID-19 pandemic, as many centres had to virtually stop or drastically reduce their transplantation programmes owing to a dramatic reduction in the number of donors and the switch of many care facilities into COVID-19 units. SARS-CoV-2 testing in donors and recipients has been implemented in many places, but the effect of COVID-19 on the outcome of liver transplantation is unknown. The role of post- transplantation immune suppression on the course of COVID-19 is also largely unknown owing to the scarcity of data. Overall, information is still limited, but it is likely that COVID-19 will result in a substantial, delayed increase in liver-related mortality that will become visible in the coming months and will substantially contribute to the overall pandemic-related mortality.

The COVID-19 pandemic will also negatively affect viral hepatitis elimination programmes. The World Health Organization has set the goal of eliminating viral hepatitis $\mathrm{B}$ and $\mathrm{C}$ as major public health threats by 2030. This aim includes reducing their incidence, prevalence, morbidity and mortality by means of prevention measures, including hepatitis B vaccination, extensive screening and improved access to care and antiviral treatments. During the COVID-19 crisis, attention has been diverted from chronic viral hepatitis, despite the fact that global mortality attributed to viral hepatitis, which has been estimated by the WHO Global Hepatitis Report 2017 to be approximately 1.5 million per year, currently remains higher than that from COVID-19, while resources for public health interventions are already shrinking. Lockdown, quarantine and social distancing, closing of harm reduction and treatment facilities, including primary care settings and general practitioners, will probably hamper the major efforts made to achieve the viral hepatitis elimination goals in many areas, further increasing indirect COVID-19-linked mortality ${ }^{10}$.

The greatest effect of COVID-19 on liver-related morbidity and mortality remains to come, as a result of the global economic crisis that has already begun. Bankruptcies, job losses, money and food shortage, social isolation and family issues will lead to increases in alcohol and drug use, whereas access to care will suffer from the collapse of health-care structures and organizations, and from government policies diverting resources elsewhere. This situation could translate into a substantial increase in blood-borne virus transmissions (in addition to the current opioid epidemic in North America, which is already rapidly increasing the incidence of new hepatitis C cases), as well as in alcoholic liver disorders and decompensations, resulting in many more patients with cirrhosis, hepatocellular carcinoma, liver transplantation and liver-related death. These unforeseen effects of the COVID-19 pandemic will take years to become visible, but they are inevitable.

In summary (BOX 1), SARS-CoV-2 infection appears to relatively spare the liver, with negligible liver-related mortality during the course of COVID-19 (with the exceptions of liver failure in patients with very severe COVID-19 infection leading to multivisceral deficiencies and of patients with decompensated cirrhosis). Nevertheless, the effect of the COVID-19 pandemic on liver-related mortality could be enormous, though hidden, delayed and not ascribed. Similar conclusions apply to many other medical specialties and should be considered when drawing up a final assessment of the COVID-19 pandemic, the policies put in place to combat it and the lessons to be learnt for the next pandemic. 
1. Qi, F., Qian, S., Zhang, S. \& Zhang, Z. Single cell RNA sequencing of 13 human tissues identify cell types and receptors of human coronaviruses. Biochem. Biophys. Res. Commun. 526, 135-140 (2020).

2. Chai, X. et al. Specific ACE2 expression in cholangiocytes may cause liver damage after 2019-nCoV infection. Preprint at bioRxiv https://doi.org/10.1101/2020.02.03.931766 (2020).

3. Cai, Q. et al. COVID-19: abnormal liver function tests. J. Hepatol. https://doi.org/10.1016/j.jhep.2020.04.006 (2020).

4. Wichmann, D. et al. Autopsy findings and venous thromboembolism in patients with COVID-19. Ann. Intern. Med. https://doi.org 10.7326/M20-2003 (2020).

5. Gao, F. et al. Metabolic associated fatty liver disease increase COVID-19 disease severity in non-diabetic patients. J. Gastroenterol. Hepatol. https://doi.org/10.1111/jgh.15112 (2020).

6. Singh, S. \& Khan, A. Clinical characteristics and outcomes of COVID-19 among patients with pre-existing liver disease in United States: a multi-center research network study. Gastroenterology https://doi.org/10.1053/j.gastro.2020.04.064 (2020).

7. Oyelade, T., Alqahtani, J. \& Canciani, G. Prognosis of COVID-19 in patients with liver and kidney diseases: an early systematic review and meta-analysis. Trop Med. Infect. Dis. 15, E80 (2020).
8. Moon, A. M. et al. High mortality rates for SARS-CoV-2 infection in patients with pre-existing chronic liver disease and cirrhosis: preliminary results from an international registry. J. Hepatol. https://doi.org/10.1016/j.jhep.2020.05.013 (2020).

9. Tapper, E. B. \& Asrani, S. K. The COVID-19 pandemic will have a long-lasting impact on the quality of cirrhosis care. J. Hepatol. https://doi.org/10.1016/j.jhep.2020.04.005 (2020).

10. Karimi-Sari, H. \& Rezaee-Zavareh, M. S. COVID-19 and viral hepatitis elimination programs: are we stepping backward? Liver Int. https://doi.org/10.1111/liv.14486 (2020).

\section{Competing interests}

The author has served as an advisor for Abbvie, Gilead, GlaxoSmithKline, Merck, Regulus and Siemens Healthcare.

\section{RELATED LINKS}

COVID-19 Dashboard by the Center for Systems Science and Engineering at Johns Hopkins University, Baltimore, Maryland: https://systems.jhu.edu/ WHO Global Hepatitis Report 2017: https://www.who.int/hepatitis/ publications/global-hepatitis-report2017/en/ 